\title{
High Complexity Bit-Plane Security Enchancement in BPCS Steganography
}

\author{
Andysah Putera Utama Siahaan \\ Faculty of Computer Science \\ Universitas Pembangunan Panca Budi \\ Jl. Jend. Gatot Subroto Km. 4,5 Sei Sikambing, \\ 20122, Medan, Sumatera Utara, Indonesia
}

\begin{abstract}
In BPCS Steganography, data hiding will be split into blocks that have a high complexity where the blocks are categorized into informative and noise-like regions. A noise-like region is a bit-plane that has the greatest probability as a data hiding since it has a high complexity. In this region, the data inserted is vulnerable to attack. Someone can easily take a series of characters that are stored on a noise-like region previously if the system is not modified. Improving the bit-plane composition is to increase data security. Bit-plane will be combined with a specified key. The key should be changed to bit-plane form as well. The key that has already been turned into the bit-plane will be mated with the original data. Using an exclusive-or of this part is the best way to produce the cipher bit-plane. Finally, the data residing on the cover image produced have a high-security level.
\end{abstract}

\section{General Terms}

Steganography, Cryptography, Security

\section{Keywords}

Bit-Plane, High Complexity, Segmentation

\section{INTRODUCTION}

Steganography is The technique to hide secret information in some other carrier without any apparent evidence of data exchange [5]. Steganography is also the art of data hiding. It covers messages in a vessel image [1][3]. Only the receiver knows what happens inside the picture. In steganography, sometimes the user do not think about the security level. When concealing the data, it is all the original data. It means those are hidden without encryption. Bit-Plane Complexity Segmentation, BPCS, is one of the steganography techniques that grouping the set of the original message to bit-planes [5]. A noise-like region consist complex pattern [11][12]. It happens since the bit-plane has the intensity variation. BPCS has an $8 \times 8$ matrix to represent the information. It serves to divide the plaintext to layers. There are totally eight layers to represent those bits in the plaintext. Complexity is the formula to measure whether the bit-plane is ready to be used. By attacking the bit-plane, someone might be retrieving the secret message. Modifying the structure of the noise-like region is the security technique to increase the security system [2]. There are numerous ways to manipulate the bit-plane. It can be combined with encryption to generate the new bit order. Creating the cipher block has numerous benefits to camouflage the original message. Once the cipher bits are created, the attacker must spare more time to resolve the message.

\section{CRYPTOGRAPHY SCHEME}

Steganography is the embedding messages art such that only the specific participants know the existence of the information [8] while cryptography is the way to change the character with the help of particular algorithms for turning the original data into an unintelligible form [7][16]. Steganography is hiding the message in a cover image so that it becomes invisible [9]. The extraction will involve the both method in retrieving the hidden information [10].

Table 1. Comparison of Steganography and Cryptography

\begin{tabular}{|l|l|l|}
\hline \multicolumn{1}{|c|}{ Criteria } & \multicolumn{1}{|c|}{ Steganography } & \multicolumn{1}{c|}{ Cryptography } \\
\hline Carrier & All digital media & $\begin{array}{l}\text { Plaintext/ } \\
\text { image/audio }\end{array}$ \\
\hline $\begin{array}{l}\text { Hiding } \\
\text { Information }\end{array}$ & Yes & No \\
\hline $\begin{array}{l}\text { Additional } \\
\text { Carrier }\end{array}$ & Required & Not required \\
\hline $\begin{array}{l}\text { Hidden } \\
\text { Message }\end{array}$ & Imperceptible & $\begin{array}{l}\text { Detection of } \\
\text { message is } \\
\text { possible }\end{array}$ \\
\hline Key & Optional & Required \\
\hline Visibility & Never visible & Always visible \\
\hline
\end{tabular}

Table 1 shows the comparison in steganography and cryptography. The criteria describe the parameter compared to both security techniques.

\section{RELATED WORK}

Eiji Kawaguchi and R. O. Eason discovered the new technique to hide the information. It is called Bit-Plane Complexity Segmentation (BPCS). The information hiding capacity is around $50 \%$ of the container size [4]. The image consists of three layers. Each layer component value ranges from zero 0 to 255 , where zero represents the darkest color and 255 represent the brightest [13]. The image of the document is divided into several segments with a size of $8 \times 8$ pixels of each segment. In the 8-bit image of the document, each segment has eight bit-planes representing the pixels of each of these bits. The process of division of segment $8 \times 8$ pixels into eight bits is called the bit slicing plane [15]. The process of inserting a message is carried on the segments that have high complexity. This is called noise-like region. In these segments, the insertion is not only performed on the least significant bit, but on the whole noise-like bit planes.

In steganography, a message is hidden inside a container such as a picture, movie or audio [14]. Some of them use BPCS algorithm to cover up the message. But it is a problem when 
the image does not fit to maintain. The data is vulnerable. There is no encryption method inserted in its algorithm.

\section{PROPOSED WORK}

The strength of BPCS depends on the sharpness of the eye can see behind the image. A subtle difference is that the color file has a slightly different file structure [6]. Usually, humankind is not able to extract the strange dot or secret information in noise-like regions. It is a complicated color intensity pattern. But, to improve the security, the noise-like pattern needs to be modified. Every message in bit-planes mates to the encryption key bit-plane. The modification is not affecting the image quality. Meanwhile, BPCS is robustness against third party attacks.

This research aims to combine the steganography and cryptography. One Time Pad is a symmetric key cryptography technique that has the same formula for encryption and decryption. The key used must be replicated as long as the bitplane. The key can be generated from the random or regular key by typing them on a keyboard. If a random key stream is used, the ciphertext will be random as well. By combining One Time Pad and BPCS will make the concealment perfect. The attacker will be deceived. He gets a set of bits obtained at the time of interception, but unfortunately, the hard work required to break the message. It makes the combination of two methods work together. The following equation shows the One Time Pad formula.

$$
C T=P T \text { xor Key }
$$

Where:

$\begin{array}{lll}\text { CT } & : & \text { Cipher Bit-Plane } \\ \text { PT } & : & \text { Plaint Bit-Plane } \\ \text { Key } & : & \text { Password Block }\end{array}$

The method is delineated in several steps as follows.

- Convert the carrier image from PBC to CGC

- Make the bit-plane

- Bit-plane analysis

- Size-estimation

- Processing message

- Encrypting message

- Implant secret blocks into carrier image.

- Save file

\section{TESTING AND EVALUATION}

The original pixels are transformed into numbers from 0 to 255 which represent the color intensity. Those are turned into pure Binary Code (PBC) system. Each row and column represent the pixel position in the image. Table 2 is an example of image segmentation. Every segment consists of 64 pixels. It consists of three layers, red, green and blue.

Table 2. An 8x8 pixel of Image Light Intensity

\begin{tabular}{|c|c|c|c|c|c|c|c|c|}
\hline \multicolumn{10}{|c|}{ PBC Pixel Segment 1} \\
\hline & 0 & 1 & 2 & 3 & 4 & 5 & 6 & 7 \\
\hline 0 & 127 & 27 & 29 & 39 & 49 & 65 & 67 & 69 \\
\hline 1 & 31 & 52 & 12 & 1 & 7 & 0 & 1 & 10 \\
\hline 2 & 23 & 29 & 24 & 28 & 37 & 44 & 41 & 21 \\
\hline 3 & 9 & 14 & 20 & 35 & 32 & 44 & 34 & 1 \\
\hline 4 & 54 & 44 & 63 & 47 & 59 & 85 & 60 & 74 \\
\hline
\end{tabular}

\begin{tabular}{|c|c|c|c|c|c|c|c|c|}
\hline 5 & 117 & 91 & 121 & 169 & 186 & 185 & 203 & 190 \\
\hline 6 & 170 & 181 & 193 & 209 & 208 & 213 & 216 & 235 \\
\hline 7 & 200 & 198 & 179 & 184 & 198 & 187 & 199 & 247 \\
\hline
\end{tabular}

Every single pixel has to be converted to binary. It will split the order of the bits into the similar bit-plane. The PBC form must be turned into CGC to remap the bit planes to perform the message insertion. It stems from the fact that CGC is better than $\mathrm{PBC}$ in producing a better-looking stego image. Table 3 and Table 4 show the comparison of PBC and GCC. The insertion in PBC is irregularities.

Table 3. Pure Binary Code Segment

\begin{tabular}{|c|c|c|c|c|c|c|c|c|}
\hline \multicolumn{7}{|c|}{ PBC (Pure Binary Code) } \\
\hline & 0 & 1 & 2 & 3 & 4 & 5 & 6 & 7 \\
\hline 0 & 01111111 & 00011011 & 00011101 & 00100111 & 00110001 & 01000001 & 01000011 & 01000101 \\
\hline 1 & 00011111 & 00110100 & 00001100 & 00000001 & 00000111 & 00000000 & 00000001 & 00001010 \\
\hline 2 & 00010111 & 00011101 & 00011000 & 00011100 & 00100101 & 00101100 & 00101001 & 00010101 \\
\hline 3 & 00001001 & 00001110 & 00010100 & 00100011 & 00100000 & 00101100 & 00100010 & 00000001 \\
\hline 4 & 00110110 & 00101100 & 00111111 & 00101111 & 00111011 & 01010101 & 00111100 & 01001010 \\
\hline 5 & 01110101 & 01011011 & 01111001 & 10101001 & 10111010 & 10111001 & 11001011 & 10111110 \\
\hline 6 & 10101010 & 10110101 & 11000001 & 11010001 & 11010000 & 11010101 & 11011000 & 11101011 \\
\hline 7 & 11001000 & 11000110 & 10110011 & 10111000 & 11000110 & 10111011 & 11000111 & 11110111 \\
\hline
\end{tabular}

Let's take an example from row 0 and column 0 . The binary digit is 01111111 . The are eight digits. As seen in the formula, the first numbers are identical while the next come from the operation of the preceding digits.

\begin{tabular}{|c|c|c|}
\hline PBC & & $0_{1} 1_{2} 1_{3} 1_{4} 1_{5} 1_{6} 1_{7} 1_{8}$ \\
\hline $\mathrm{CGC}_{1}$ & : & $\begin{array}{l}\mathrm{PBC}_{1} \\
0\end{array}$ \\
\hline $\mathrm{CGC}_{2}$ & : & $\begin{array}{l}\mathrm{PBC}_{2} \oplus \mathrm{PBC}_{1} \\
1 \oplus 0 \\
1\end{array}$ \\
\hline $\mathrm{CGC}_{3}$ & . & $\begin{array}{l}\mathrm{PBC}_{3} \oplus \mathrm{PBC}_{2} \\
1 \oplus 1 \\
0\end{array}$ \\
\hline $\mathrm{CGC}_{4}$ & : & $\begin{array}{l}\mathrm{PBC}_{4} \oplus \mathrm{PBC}_{3} \\
1 \oplus 1 \\
0\end{array}$ \\
\hline $\mathrm{CGC}_{5}$ & : & $\begin{array}{l}\mathrm{PBC}_{5} \oplus \mathrm{PBC}_{4} \\
1 \oplus 1 \\
0\end{array}$ \\
\hline $\mathrm{CGC}_{6}$ & . & $\begin{array}{l}\mathrm{PBC}_{6} \oplus \mathrm{PBC}_{5} \\
1 \oplus 1 \\
0\end{array}$ \\
\hline $\mathrm{CGC}_{7}$ & : & $\begin{array}{l}\mathrm{PBC}_{7} \oplus \mathrm{PBC}_{6} \\
1 \oplus 1 \\
0\end{array}$ \\
\hline CGC & . & $\begin{array}{l}\mathrm{PBC}_{8} \oplus \mathrm{PBC}_{7} \\
1 \oplus 1 \\
0\end{array}$ \\
\hline
\end{tabular}

The CGC is 01000000 . This calculation continues until 64 times for each segment. Table 4 is the result of the first segment conversion and it is repeated until all the segments are finished.

Table 4. Canonical Gray Code Segment

\begin{tabular}{|c|c|c|c|c|c|c|c|c|}
\hline \multicolumn{10}{|c|}{ CGC (Canonical Gray Code) } \\
\hline & 0 & 1 & 2 & 3 & 4 & 5 & 6 & 7 \\
\hline 0 & 01000000 & 00010110 & 00010011 & 00110100 & 00101001 & 01100001 & 01100010 & 01100111 \\
\hline 1 & 00010000 & 00101110 & 00001010 & 00000001 & 00000100 & 00000000 & 00000001 & 00001111 \\
\hline
\end{tabular}




\begin{tabular}{|l|l|l|l|l|l|l|l|l|}
\hline 2 & 00011100 & 00010011 & 00010100 & 00010010 & 00110111 & 00111010 & 00111101 & 00011111 \\
\hline 3 & 00001101 & 00001001 & 00011110 & 00110010 & 00110000 & 00111010 & 00110011 & 00000001 \\
\hline 4 & 00101101 & 00111010 & 00100000 & 00111000 & 00100110 & 01111111 & 00100010 & 01101111 \\
\hline 5 & 01001111 & 01110110 & 01000101 & 11111101 & 11100111 & 11100101 & 10101110 & 11100001 \\
\hline 6 & 1111111 & 11101111 & 10100001 & 10111001 & 10111000 & 10111111 & 10110100 & 10011110 \\
\hline 7 & 10101100 & 10100101 & 11101010 & 11100100 & 10100101 & 11100110 & 10100100 & 10001100 \\
\hline
\end{tabular}

Every bit order in Canonical Gray Code is split into bitplanes. For example, on the first bit-plane, it is resulted from drawing every first bit on CGC map while on the last one, it is from the last bit. Embedding a file-block in an n-th least PBC plane means modifying the colors of several pixels in that block with the value $2^{\mathrm{n}-1}$. In this case the "blocking effect" likely to appear on the stego image. While in the CGC embedding, the color change "differs pixel by pixel" in the block varying from 1 to $2^{\mathrm{n}-1}$. The average change in the block is $2^{\text {n- }-1}$. Table 5 is the result of splitting bit-planes.

Table 5. Bit-Planes Slicing

\begin{tabular}{|c|c|c|c|c|c|c|c|c|}
\hline \multicolumn{3}{|c|}{ Bit Plane 1} & \multicolumn{4}{c|}{9} & \multicolumn{3}{c|}{0,080357143} \\
\hline & 0 & 1 & 2 & 3 & 4 & 5 & 6 & 7 \\
\hline 0 & 0 & 0 & 0 & 0 & 0 & 0 & 0 & 0 \\
\hline 1 & 0 & 0 & 0 & 0 & 0 & 0 & 0 & 0 \\
\hline 2 & 0 & 0 & 0 & 0 & 0 & 0 & 0 & 0 \\
\hline 3 & 0 & 0 & 0 & 0 & 0 & 0 & 0 & 0 \\
\hline 4 & 0 & 0 & 0 & 0 & 0 & 0 & 0 & 0 \\
\hline 5 & 0 & 0 & 0 & 1 & 1 & 1 & 1 & 1 \\
\hline 6 & 1 & 1 & 1 & 1 & 1 & 1 & 1 & 1 \\
\hline 7 & 1 & 1 & 1 & 1 & 1 & 1 & 1 & 1 \\
\hline
\end{tabular}

\begin{tabular}{|c|c|c|c|c|c|c|c|c|}
\hline \multicolumn{5}{|c|}{ Bit Plane 2} & 33 & \multicolumn{3}{|c|}{0,294642857} \\
\hline & 0 & 1 & 2 & 3 & 4 & 5 & 6 & 7 \\
\hline 0 & 1 & 0 & 0 & 0 & 0 & 1 & 1 & 1 \\
\hline 1 & 0 & 0 & 0 & 0 & 0 & 0 & 0 & 0 \\
\hline 2 & 0 & 0 & 0 & 0 & 0 & 0 & 0 & 0 \\
\hline 3 & 0 & 0 & 0 & 0 & 0 & 0 & 0 & 0 \\
\hline 4 & 0 & 0 & 0 & 0 & 0 & 1 & 0 & 1 \\
\hline 5 & 1 & 1 & 1 & 1 & 1 & 1 & 0 & 1 \\
\hline 6 & 1 & 1 & 0 & 0 & 0 & 0 & 0 & 0 \\
\hline 7 & 0 & 0 & 1 & 1 & 0 & 1 & 0 & 0 \\
\hline
\end{tabular}

\begin{tabular}{|c|c|c|c|c|c|c|c|c|}
\hline \multicolumn{5}{|c|}{ Bit Plane 3} & 34 & \multicolumn{3}{|c|}{0,303571429} \\
\hline & 0 & 1 & 2 & 3 & 4 & 5 & 6 & 7 \\
\hline 0 & 0 & 0 & 0 & 1 & 1 & 1 & 1 & 1 \\
\hline 1 & 0 & 1 & 0 & 0 & 0 & 0 & 0 & 0 \\
\hline 2 & 0 & 0 & 0 & 0 & 1 & 1 & 1 & 0 \\
\hline 3 & 0 & 0 & 0 & 1 & 1 & 1 & 1 & 0 \\
\hline 4 & 1 & 1 & 1 & 1 & 1 & 1 & 1 & 1 \\
\hline 5 & 0 & 1 & 0 & 1 & 1 & 1 & 1 & 1 \\
\hline 6 & 1 & 1 & 1 & 1 & 1 & 1 & 1 & 0 \\
\hline 7 & 1 & 1 & 1 & 1 & 1 & 1 & 1 & 0 \\
\hline
\end{tabular}

\begin{tabular}{|l|c|c|c|c|c|c|c|c|}
\hline \multicolumn{4}{|c|}{ Bit Plane 4 } & 48 & \multicolumn{3}{|c|}{0,428571429} \\
\hline & 0 & 1 & 2 & 3 & 4 & 5 & 6 & 7 \\
\hline 0 & 0 & 1 & 1 & 1 & 0 & 0 & 0 & 0 \\
\hline 1 & 1 & 0 & 0 & 0 & 0 & 0 & 0 & 0 \\
\hline 2 & 1 & 1 & 1 & 1 & 1 & 1 & 1 & 1 \\
\hline 3 & 0 & 0 & 1 & 1 & 1 & 1 & 1 & 0 \\
\hline 4 & 0 & 1 & 0 & 1 & 0 & 1 & 0 & 0 \\
\hline
\end{tabular}

\begin{tabular}{|l|l|l|l|l|l|l|l|l|}
5 & 0 & 1 & 0 & 1 & 0 & 0 & 0 & 0 \\
\hline 6 & 1 & 0 & 0 & 1 & 1 & 1 & 1 & 1 \\
\hline 7 & 0 & 0 & 0 & 0 & 0 & 0 & 0 & 0 \\
\hline
\end{tabular}

\begin{tabular}{|c|c|c|c|c|c|c|c|c|}
\hline \multicolumn{5}{|c|}{ Bit Plane 5 } & 61 & \multicolumn{3}{|c|}{0,544642857} \\
\hline & 0 & 1 & 2 & 3 & 4 & 5 & 6 & 7 \\
\hline 0 & 0 & 0 & 0 & 0 & 1 & 0 & 0 & 0 \\
\hline 1 & 0 & 1 & 1 & 0 & 0 & 0 & 0 & 1 \\
\hline 2 & 1 & 0 & 0 & 0 & 0 & 1 & 1 & 1 \\
\hline 3 & 1 & 1 & 1 & 0 & 0 & 1 & 0 & 0 \\
\hline 4 & 1 & 1 & 0 & 1 & 0 & 1 & 0 & 1 \\
\hline 5 & 1 & 0 & 0 & 1 & 0 & 0 & 1 & 0 \\
\hline 6 & 1 & 1 & 0 & 1 & 1 & 1 & 0 & 1 \\
\hline 7 & 1 & 0 & 1 & 0 & 0 & 0 & 0 & 1 \\
\hline
\end{tabular}

\begin{tabular}{|l|l|l|l|l|l|l|l|l|}
\hline \multicolumn{5}{|c|}{ Bit Plane 6 } & 52 & \multicolumn{3}{|c|}{0,464285714} \\
\hline & 0 & 1 & 2 & 3 & 4 & 5 & 6 & 7 \\
\hline 0 & 0 & 1 & 0 & 1 & 0 & 0 & 0 & 1 \\
\hline 1 & 0 & 1 & 0 & 0 & 1 & 0 & 0 & 1 \\
\hline 2 & 1 & 0 & 1 & 0 & 1 & 0 & 1 & 1 \\
\hline 3 & 1 & 0 & 1 & 0 & 0 & 0 & 0 & 0 \\
\hline 4 & 1 & 0 & 0 & 0 & 1 & 1 & 0 & 1 \\
\hline 5 & 1 & 1 & 1 & 1 & 1 & 1 & 1 & 0 \\
\hline 6 & 1 & 1 & 0 & 0 & 0 & 1 & 1 & 1 \\
\hline 7 & 1 & 1 & 0 & 1 & 1 & 1 & 1 & 1 \\
\hline
\end{tabular}

\begin{tabular}{|c|c|c|c|c|c|c|c|c|}
\hline \multicolumn{5}{|c|}{ Bit Plane 7} & 5 & \multicolumn{3}{|c|}{0,508928571} \\
\hline & 0 & 1 & 2 & 3 & 4 & 5 & 6 & 7 \\
\hline 0 & 0 & 1 & 1 & 0 & 0 & 0 & 1 & 1 \\
\hline 1 & 0 & 1 & 1 & 0 & 0 & 0 & 0 & 1 \\
\hline 2 & 0 & 1 & 0 & 1 & 1 & 1 & 0 & 1 \\
\hline 3 & 0 & 0 & 1 & 1 & 0 & 1 & 1 & 0 \\
\hline 4 & 0 & 1 & 0 & 0 & 1 & 1 & 1 & 1 \\
\hline 5 & 1 & 1 & 0 & 0 & 1 & 0 & 1 & 0 \\
\hline 6 & 1 & 1 & 0 & 0 & 0 & 1 & 0 & 1 \\
\hline 7 & 0 & 0 & 1 & 0 & 0 & 1 & 0 & 0 \\
\hline
\end{tabular}

\begin{tabular}{|c|c|c|c|c|c|c|c|c|}
\hline \multicolumn{7}{|c|}{ Bit Plane 8 } & 56 & \multicolumn{3}{|c|}{0,5} \\
\hline & 0 & 1 & 2 & 3 & 4 & 5 & 6 & 7 \\
\hline 0 & 0 & 0 & 1 & 0 & 1 & 1 & 0 & 1 \\
\hline 1 & 0 & 0 & 0 & 1 & 0 & 0 & 1 & 1 \\
\hline 2 & 0 & 1 & 0 & 0 & 1 & 0 & 1 & 1 \\
\hline 3 & 1 & 1 & 0 & 0 & 0 & 0 & 1 & 1 \\
\hline 4 & 1 & 0 & 0 & 0 & 0 & 1 & 0 & 1 \\
\hline 5 & 1 & 0 & 1 & 1 & 1 & 1 & 0 & 1 \\
\hline 6 & 1 & 1 & 1 & 1 & 0 & 1 & 0 & 0 \\
\hline 7 & 0 & 1 & 0 & 0 & 1 & 0 & 0 & 0 \\
\hline
\end{tabular}

Every bit-plane has the complexity value. The threshold is the limit to determine whether the bit-plane can be inserted by the message. The complexity is measured by how many times the bit change from 0 to 1 and from 1 to 0 . The maximal bit change value of $8 \times 8$ block is 112 . The formula to calculate the complexity is given in equation 2 . 


$$
\alpha=\frac{k}{n}
$$

Where:

$$
\begin{array}{ll}
\alpha & : \text { Bit-Plane Complexity } \\
\mathrm{k} & : \text { Total Bit change } \\
\mathrm{n} & : \text { Maximum Change of } 8 \times 8 \text { bit- }
\end{array}
$$
plane

Table 6 shows the complexity of all bit-planes. The next procedure is to set the limitation between informative and noise-like region. The limit is called threshold. Generally, the standard value of the threshold is 0.3 , but it can be modified as needed. The modification is often performed to avoid the informative area or to add the noise-like area. The $n$ value is set to the maximum that is 112 .

Table 6. The Complexity of Bit-Planes

\begin{tabular}{|r|r|c|}
\hline \multicolumn{1}{|c|}{ Bit-Plane } & \multicolumn{1}{|c|}{ Bit Change } & Complexity \\
\hline 1 & 9 & 0,080357142857 \\
\hline 2 & 33 & 0,294642857143 \\
\hline 3 & 34 & 0,303571428571 \\
\hline 4 & 48 & 0,428571428571 \\
\hline 5 & 61 & 0,544642857143 \\
\hline 6 & 52 & 0,464285714286 \\
\hline 7 & 57 & 0,508928571429 \\
\hline 8 & 56 & 0,500000000000 \\
\hline
\end{tabular}

Assume the threshold limit is 0.3 . In Table 6, there are two numbers are below the threshold. They are bit-plane number 1 and 2. The numbers which are in that range are called the informative regions. The message cannot insert these regions. Putting the message block in these ranges will make the stego image easily distinguished. The process of embedding is skipped to the next bit-plane.

In this case, the word to embed is "ANDYSAH!". The word consists of eight characters. It has one message block. Table 6 and Table 8 show the plain message and the bit-planes.

Table 7. The Original Message

\begin{tabular}{|c|c|c|}
\hline Char & Dec. & Biner \\
\hline $\mathrm{A}$ & 65 & 1000001 \\
\hline $\mathrm{N}$ & 78 & 1001110 \\
\hline $\mathrm{D}$ & 68 & 1000100 \\
\hline $\mathrm{Y}$ & 89 & 1011001 \\
\hline $\mathrm{S}$ & 83 & 1010011 \\
\hline $\mathrm{A}$ & 65 & 1000001 \\
\hline $\mathrm{H}$ & 72 & 1001000 \\
\hline ! & 33 & 100001 \\
\hline
\end{tabular}

Table 8. The Bit-Plane of Message

\begin{tabular}{|c|c|c|c|c|c|c|c|c|}
\hline \multicolumn{10}{|c|}{ Bit Plane Message } \\
\hline & 0 & 1 & 2 & 3 & 4 & 5 & 6 & 7 \\
\hline 0 & 0 & 1 & 0 & 0 & 0 & 0 & 0 & 1 \\
\hline 1 & 0 & 1 & 0 & 0 & 1 & 1 & 1 & 0 \\
\hline 2 & 0 & 1 & 0 & 0 & 0 & 1 & 0 & 0 \\
\hline 3 & 0 & 1 & 0 & 1 & 1 & 0 & 0 & 1 \\
\hline 4 & 0 & 1 & 0 & 1 & 0 & 0 & 1 & 1 \\
\hline 5 & 0 & 1 & 0 & 0 & 0 & 0 & 0 & 1 \\
\hline 6 & 0 & 1 & 0 & 0 & 1 & 0 & 0 & 0 \\
\hline 7 & 0 & 0 & 1 & 0 & 0 & 0 & 0 & 1 \\
\hline
\end{tabular}

The bit-plane must be transformed into encrypted block before it is completely embedded in the noise-like regions. This step is the main part of the study. The encryption method takes apart in this step. The One Time Pad is the very common algorithm to be applied in BPCS. The OTP needs key as the password to produce the cipher bit-planes. For example, the key is "SDM21". The word is repeated until the length of bit-plane is covered.

Table 9. Repeated Key

\begin{tabular}{|c|c|c|}
\hline Char & Dec. & Biner \\
\hline S & 83 & 01010011 \\
\hline D & 68 & 01000100 \\
\hline M & 77 & 01001101 \\
\hline 2 & 50 & 00110010 \\
\hline 1 & 49 & 00110001 \\
\hline S & 83 & 01010011 \\
\hline D & 68 & 01000100 \\
\hline M & 77 & 01001101 \\
\hline
\end{tabular}

The key itself is converted to bit-plane model as shown in Table 10. The key can replace the conjugation block. BPCS uses conjugation block to converted informative region to noise-like region. It is needed when the informative region is found. In conventional BPCS, not all the bit-plane needs to be conjugated. But in combining with the encryption, mating the bit-plane with the conjugation block is a must.

\section{Table 10. Bit-Plane of the Key}

\begin{tabular}{|c|c|c|c|c|c|c|c|c|}
\hline \multicolumn{10}{|c|}{ Bit Plane Message } \\
\hline & 0 & 1 & 2 & 3 & 4 & 5 & 6 & 7 \\
\hline 0 & 0 & 1 & 0 & 1 & 0 & 0 & 1 & 1 \\
\hline 1 & 0 & 1 & 0 & 0 & 0 & 1 & 0 & 0 \\
\hline 2 & 0 & 1 & 0 & 0 & 1 & 1 & 0 & 1 \\
\hline 3 & 0 & 0 & 1 & 1 & 0 & 0 & 1 & 0 \\
\hline 4 & 0 & 0 & 1 & 1 & 0 & 0 & 0 & 1 \\
\hline 5 & 0 & 1 & 0 & 1 & 0 & 0 & 1 & 1 \\
\hline 6 & 0 & 1 & 0 & 0 & 0 & 1 & 0 & 0 \\
\hline 7 & 0 & 1 & 0 & 0 & 1 & 1 & 0 & 1 \\
\hline
\end{tabular}

The bit-plane of message and key must be transformed by performing the exclusive-or $(\oplus)$. The result of the bit-planes is the cipher bit-planes. After all the bit-planes are encrypted, the bit-planes are returned to its original position and reconverted to pure binary code before they are finally rewritten to the new stego image.

Table 11. Bit-Plane of Cipher

\begin{tabular}{|c|c|c|c|c|c|c|c|c|}
\hline \multicolumn{10}{|c|}{ Cipher Bit-Plane } \\
\hline & 0 & 1 & 2 & 3 & 4 & 5 & 6 & 7 \\
\hline 0 & 0 & 0 & 0 & 1 & 0 & 0 & 1 & 0 \\
\hline 1 & 0 & 0 & 0 & 0 & 1 & 0 & 1 & 0 \\
\hline 2 & 0 & 0 & 0 & 0 & 1 & 0 & 0 & 1 \\
\hline 3 & 0 & 1 & 1 & 0 & 1 & 0 & 1 & 1 \\
\hline 4 & 0 & 1 & 1 & 0 & 0 & 0 & 1 & 0 \\
\hline 5 & 0 & 0 & 0 & 1 & 0 & 0 & 1 & 0 \\
\hline 6 & 0 & 0 & 0 & 0 & 1 & 1 & 0 & 0 \\
\hline 7 & 0 & 1 & 1 & 0 & 1 & 1 & 0 & 0 \\
\hline
\end{tabular}


Table 11 shows the bit-plane after encryption and Table 12 shows the bit-plane after conversion. The previous process turns the message into the encrypted message.

Table 12. Cipher Message

\begin{tabular}{|c|c|c|}
\hline Char & Dec. & Biner \\
\hline & 18 & 00010010 \\
\hline & 10 & 00001010 \\
\hline & 9 & 00001001 \\
\hline $\mathrm{k}$ & 107 & 01101011 \\
\hline $\mathrm{b}$ & 98 & 01100010 \\
\hline & 18 & 00010010 \\
\hline & & 00001100 \\
\hline & 12 & \\
\hline $\mathrm{l}$ & 108 & 01101100 \\
\hline
\end{tabular}

As discussed earlier, the bit-plane 1 and 2 in Table 5 are the informative regions. The embedding starts from the third bitplane which the complexity value is 0,303571428571 . This value is greater than the threshold, so it is safe to hide. After the calculation is finished, the third bit-plane is now replaced by the message block. This process is to make the intruder takes more time to break the message. Once the intruders solve the bit-plane, they will get the key to reconstructing the bit-planes into the plain text back.

\section{ANALYSIS}

The different before and after hiding can be calculated by Peak Signal to Noise Ratio. It shows how close the vessel image compared to the original image. The closer the distance, the better the result. The following formula shows how it works.

$$
P S N R=20 \log \left(\frac{255}{\sqrt{\frac{1}{n} * \sum_{i=0}^{n-1}(D-S)^{2}}}\right)
$$

\section{CONCLUSION}

BPCS Steganography uses the noise-like region to store the information which can be associated with encryption. The noise-like pattern can be changed without changing the original information. One Time Pad is the best way of applying the security technique in this method since it does not use the complicated calculation. It is only to reconstruct the message block with the exclusive-or operation by providing the key as the password. The strength key ensures the security level increase.

\section{FUTURE SCOPE}

The threshold needs to limit the hiding into the bit-planes. It is still static. The future research is expected that the vessel image is smoother than the earlier. The hiding can be placed based on a specific threshold value. The value itself is adjustable. The threshold is automatically determined by image analysis module. They will be different in the different case. The program will find the highest possibility complexity. Hence, it needs an appropriate algorithm to distinguish the threshold value. Afterward, the container will be undetected by human eyes. The vessel image after embedding is a lossy steganography. The method can be further developed to return the original bits when extraction.

\section{REFERENCES}

[1] N. Johnson dan S. Jajodia, "Exploring Steganography: Seeing The Unseen," IEEE Computer, pp. 26-34, 1998.
[2] Y. K. S. P. S. M. M. B. Prashant Lahane, "Visual Cryptography and BPCS Steganography for Data Shielding," International Journal Of Engineering And Computer Science, vol. 4, no. 5, pp. 11997-11999, 2015.

[3] P. R. Rudramath dan M. R. Madki, "High Capacity Data Embedding Technique Using Improved BPCS Steganography," International Journal of Scientific and Research Publications, vol. 2, no. 7, pp. 1-4, 2012.

[4] E. Kawaguchi dan R. O. Eason, "Principle and applications of BPCS-Steganography," SPIE.

[5] S. Mehta, K. Dighe, M. Jagtap dan A. Ekre, "Web Based BPCS Steganography," International Journal of Computer Technology and Electronics Engineering, vol. 2, no. 2, pp. 126-130, 2015.

[6] P. P. Khairnar dan P. V. S. Ubale, "Steganography Using BPCS technology," International Journal of Engineering and Science, vol. 3, no. 2, pp. 8-16, 2013.

[7] C. Jain, VivekParate, A. Dhamanikar dan R. Badgujar, "Review on Steganography and BPCS Technology in Steganography for Increasing Data Embedding Capacity," International Journal of Innovative Research in Computer and Communication Engineering, vol. 3, no. 1, pp. 60-65, 2015.

[8] N. F. Johnson dan S. Jajodia, "Steganography: Seeing the Unseen,” IEEE Computer, pp. 26-34, 1998.

[9] B. A. Forouzan, Cryptography \& Network Security, New Delhi: McGraw Hill Publication, 2008.

[10] A.-M. A., Steganography-Based Secret And Reliable Communications Improving Steganographic Capacity And Imperceptibility, School of Information Systems, Computing and Mathematics, 2010.

[11] V. J. Patel dan N. RipalSoni, "Uncompressed Image Steganography using BPCS: Survey and Analysis," OSR Journal of Computer Engineering, vol. 15, no. 4, pp. 5764, 2013.

[12] H. Noda, M. Niimi dan EijiKawguchi, “A Steganography Based on Region Segmentation by Using Complexity Measure," Trans. of IEICE, vol. 81, no. 2, pp. 11321140, 1998.

[13] P. R. Rudramath dan M. R. Madki, "Improved BPCS Steganography Based Novel Approach for Data Embedding," International Journal of Engineering and Innovative Technology, vol. 1, no. 3, pp. 156-159, 2012.

[14] V. J. Deshmukh dan D. A. S. Alvi, "BPCS Steganography and Visual Cryptography: An Advance Technique for Online Payment Security in E-Commerce for Developing Countries," International Journal of Science and Research, vol. 4, no. 3, pp. 294-297, 2013.

[15] S. S. Solanke dan P. D. C. Dhanwani, "Survey on Modified BPCS Steganography Based on Sequence of Cipher Bits," International Journal of Engineering Development and Research, vol. 3, no. 2, pp. 1036-1040, 2015.

[16] A.P. U. Siahaan, "RC4 Technique in Visual Cryptography RGB Image Encryption," SSRG International Journal of Computer Science and Engineering (SSRG-IJCSE), vol. 3, no. 7, pp. 1-6, 2016. 


\section{AUTHOR PROFILE}

Andysah Putera Utama Siahaan was born in Medan, Indonesia, in 1980. He received the S.Kom. degree in computer science from Universitas Pembangunan Panca Budi, Medan, Indonesia, in 2010, and the M.Kom. in computer science as well from the University of Sumatera Utara, Medan, Indonesia, in 2012. In 2010, he joined the Department of Engineering, Universitas Pembangunan Panca Budi, as a Lecturer, and in 2012 became a junior researcher. $\mathrm{He}$ is applying for his $\mathrm{Ph}$. D. degree in 2016. He has written in several international journals $\mathrm{He}$ is now active in writing papers and joining conferences. 